\title{
Code of Practice for Research Data Usage Metrics Release 1
}

Martin Fenner ${ }^{1}$, Daniella Lowenberg ${ }^{2}$, Matt Jones ${ }^{3}$, Paul Needham ${ }^{4}$, Dave Vieglais ${ }^{5}$, Stephen Abrams $^{2}$, Patricia Cruse ${ }^{1}$ and John Chodacki ${ }^{2}$ on behalf of the Make Date Count Project

${ }^{1}$ DataCite, Hannover, Germany

${ }^{2}$ California Digital Library, Oakland, CA, USA

${ }^{3}$ National Center for Ecological Analysis and Synthesis, Santa Barbara, CA, USA

${ }^{4}$ Cranfield University, Cranfield, United Kingdom

${ }^{5}$ Biodiversity Research Institute, University of Kansas, Lawrence, KS, USA

Corresponding author:

Martin Fenner ${ }^{1}$, email: mfenner@datacite.org

Funding Disclosure:

This work was funded by a grant from the Alfred P. Sloan Foundation. This funding body had no role in the design of the report, or in writing the manuscript.

\section{Abstract}

The Code of Practice for Research Data Usage Metrics standardizes the generation and distribution of usage metrics for research data, enabling for the first time the consistent and credible reporting of research data usage. This is the first release of the Code of Practice and the recommendations are aligned as much as possible with the COUNTER Code of Practice Release 5 that standardizes usage metrics for many scholarly resources, including journals and books. With the Code of Practice for Research Data Usage Metrics data repositories and platform providers can report usage metrics following common best practices and using a standard report format. This is an essential step towards realizing usage metrics as a critical component in our understanding of how publicly available research data are being reused. This complements ongoing work on establishing best practices and services for data citation. 


\section{Executive Summary}

Usage metrics for research data are seen as an important indicator of impact by researchers and other stakeholders (Costas, Meijer, Zahedi, \& Wouters, 2013, Kratz \& Strasser, 2015), second only to data citations. They currently can't fill that role due to the lack of standardization on how usage metrics should be collected and reported.

The Code of Practice for Research Data Usage Metrics standardizes the generation and distribution of usage metrics for research data. This enables data repositories and platform providers to produce consistent and credible usage metrics for research data, and helps data repositories, libraries, funders and other stakeholders to understand and demonstrate the reuse of research data.

This is the first release of the Code of Practice for Research Data Usage Metrics. The recommendations are aligned as much as possible with the COUNTER Code of Practice Release 5 (COUNTER Code of Practice Release 5, 2017) that standardizes usage metrics for many scholarly resources, including journals and books. Many definitions, processing rules, and reporting recommendations apply to research data in the same way as they apply to other scholarly resources.

The dataset (a collection of data published or curated by a single agent) is the content item for which we report usage in terms of investigations (i.e. how many times metadata are accessed) and requests (i.e. how many times data are retrieved, a subset of all investigations).

Investigations and requests for components of the dataset can be reported in the same way as other scholarly resources under COUNTER Code of Practice Release 5, in that the total number of investigations or requests are summed across the components of a given dataset. Sessions allow the differentiation between total investigations and requests of a dataset (in which all accesses are summed) and unique investigations and requests (in which accesses are only counted once per dataset if they are within a unique user-session), aligned with the reporting for content items in COUNTER Code of Practice Release 5.

Some aspects of the processing and reporting of usage data are unique to research data, and the Code of Practice for Research Data Usage Metrics thus at times needs to deviate from the COUNTER Code of Practice Release 5 and specifically address them. This starts with the main use cases for data usage metrics reporting: subscription access to research data is uncommon, therefore breaking down the usage data by institution accessing the research data is less relevant. 
While there is interest in understanding the geographic distribution of investigations and requests to research data, these usage data can be reported at a coarser granularity (by country rather than by institution) and can be aggregated and openly shared.

COUNTER Code of Practice Release 5 focusses usage reporting on human users and filters out all known robots, crawlers, and spiders. While the same exclusion list should be applied to research data, there is significant legitimate usage in which humans employ scripts and other automated tools in the normal course of research. The Code of Practice for Research Data Usage Metrics defines how usage metrics from these automated tools used can be reported. Versioning is much more common and complex with research data compared to most other scholarly resources, and the Code of Practice for Research Data Usage Metrics addresses this. We recommend reporting usage metrics for each specific version, as well as the combined usage for all versions. This first release of the Code of Practice for Research Data Usage Metrics will not fully address the particular challenges associated with reporting usage for dynamically changing datasets.

Research data can be retrieved in a wide variety of file formats, different from text-based scholarly resources. For the Code of Practice for Research Data Usage Metrics we will not break down requests by file format. We will include the data volume transferred as part of the reporting, since the variations are much greater than for other scholarly resources. Reporting request data transfer volume in addition to the number of requests and investigations also helps with understanding differences between data repositories with regards to how data are packaged and made available for retrieval.

The Code of Practice for Research Data Usage Metrics enables the reporting of usage metrics by different data repositories following common best practices, and thus is an essential step towards realizing usage metrics to facilitate understanding how publicly available research datas are being reused. This complements ongoing work on establishing best practices and services for data citation (Burton, Fenner, Haak, \& Manghi, 2017). 


\section{Conventions}

This Code of Practice for Research Data is implemented using the following convention:

The keywords “MUST”, “MUST NOT”, “REQUIRED”, "RECOMMENDED”, and "OPTIONAL" in this document are to be interpreted as described in RFC 2119 ("RFC 2119:

Key words for use in RFCs to Indicate Requirement Levels," 1997).

Note that the force of these words is modified by the requirement level of the document in which they are used.

1. "MUST" (or "REQUIRED") means that the definition is an absolute requirement of the specification.

2. "MUST NOT" means that the definition is an absolute prohibition of the specification.

3. "RECOMMENDED" means that there may be valid reasons in certain circumstances to ignore a particular item, but the full implications should be understood and carefully weighed before choosing a different course.

4. "NOT RECOMMENDED" means that there may be valid reasons in certain circumstances when the particular behaviour is acceptable or even useful, but the full implications should be understood, and the case carefully weighed before implementing any behavior described with this label.

Content providers implementing the Code of Practice for Research Data Usage Metrics who feel they have a valid disagreement with a requirement of the code are requested to contact the authors and ask for clarification on interpretation of the code.

Terms appearing in italics represent variables that will be replaced with appropriate values at implementation time, for example "Error_Number : Error_Description" might resolve to "3040 : Partial Usage Available”. 


\section{Introduction}

This is the first version of a Code of Practice for Research Data. The purpose of this report is to enable data repositories and platform providers to produce consistent, comparable, and credible usage metrics for research data. This first release of the Code of Practice for Research Data Usage Metrics has been kept intentionally narrow in scope to focus on the dataset level and avoid creating unnecessary hurdles to adoption.

\subsection{General Information}

\subsubsection{Purpose}

The purpose of the Code of Practice for Research Data Usage Metrics is to facilitate the recording, exchange, and interpretation of online usage data by establishing open standards and protocols for the provision of content-provider-generated usage statistics that are consistent, comparable, and credible.

\subsubsection{Scope}

This Code of Practice for Research Data Usage Metrics is aligned with the COUNTER Code of Practice Release 5 and provides a framework for recording and exchanging online usage statistics for research data at an international level. It covers the following areas: data elements to be measured; definitions of these data elements; content and format of usage reports; requirements for data processing; and guidelines to avoid duplicate counting.

\subsubsection{Relationship to COUNTER Code of Practice Release 5}

Developed by members from the research data management community (RDM) in close coordination with COUNTER, this Code of Practice for Research Data follows the COUNTER Code of Practice Release 5 (COUNTER Code of Practice Release 5, 2017) recommendations as much as possible (where relevant) and deviates from them only when necessary. There are different use cases and practices between research data and the majority of scholarly resources. For example, research data does not need to be reported at the institutional level, but geographic aggregation may be important. Another significant difference is the need for aggregation of usage across components for all versions of a dataset. It is common practice for research data to be versioned, and we recommend reporting the usage data for each specific version and the combined usage for all versions. 
The first release of the Code of Practice for Research Data Usage Metrics only describes reporting of usage at the dataset level. For future releases, reporting usage statistics for dataset components will be considered based on community feedback. Following the COUNTER Code of Practice Release 5, standard usage statistics are not reported by format distribution, e.g., no separate numbers for downloads in CSV and XLSX formats.

Download volume (i.e., file size) can be reported. There are widely varying practices in the research data community regarding the granularity and structure of datasets, components, and collections. Reporting download volume makes it easier to compare usage for research data packaged into datasets with different granularity.

Geolocation information and country are reported, but not IP addresses. For large countries (e.g. United States) reporting at the state or province level may be enabled. Reporting of geolocation information helps to better understand usage for the same datasets hosted in multiple locations, and for datasets where usage is dependent upon the location of the user, e.g., datasets describing research in a particular geolocation.

Usage metrics are reported for each specific version of a dataset, as well as the combined usage for all versions. Usage metrics are only reported for individual datasets. In this version of the Code of Practice for Research Data Usage Metrics the is no report format for reporting usage for collections of datasets, for example all datasets in a data repository.

\subsubsection{Strategy}

The Code of Practice for Research Data Usage Metrics will evolve in response to the demands of the international library, data management, and content provider communities. The Code of Practice for Research Data Usage Metrics is continually under review; feedback on its scope and application are actively sought from all interested parties.

\subsubsection{Governance}

The Code of Practice for Research Data Usage Metrics is developed by the Make Data Count project (Make Data Count, 2017), in close collaboration with Counter Online Metrics (COUNTER) (Project COUNTER, 2002), a non-profit organization that maintains the COUNTER Code of Practice. 


\subsubsection{Definitions}

This Code of Practice for Research Data Usage Metrics provides definitions of data elements and other terms that are relevant not only to the usage reports specified in this document, but also to other reports that content providers may wish to generate. Every effort has been made to use existing COUNTER, ISO, NISO, etc. definitions where appropriate, and these sources are cited (see References and Appendix A). The following key definitions are used by the Code of Practice for Research Data Usage Metrics:

- Dataset: An aggregation of data, published or curated by a single agent, and available for access or download in one or more formats, with accompanying metadata (Dekkers \& Isaac, 2018). A dataset is a subtype of a COUNTER content item. Synonymous term: data package.

- Component: Part of the data available for a dataset that can be accessed or downloaded individually. Aligns with a COUNTER component. Synonymous terms: data file, data granule.

- Collection: A curated aggregation of datasets. Related terms: catalog, repository.

- Version: Multiple versions of a dataset are defined as significant changes to the content and/or metadata, associated with changes in one or more components, and that would result in changes to fixity attributes of the components.

\subsubsection{Versions}

The Code of Practice for Research Data Usage Metrics will be extended and upgraded as necessary, based on input from the communities it serves. Future versions might be integrated into the COUNTER Code of Practice. A continuous maintenance process will allow the Code of Practice for Research Data Usage Metrics to evolve over time minimizing the need for major version changes.

\subsubsection{Auditing and Code of Practice for Research Data Usage Metrics Compliance} No content provider following the Code of Practice for Research Data Usage Metrics has been audited at the time of this first release of the Code of Practice for Research Data Usage Metrics. While we expect the auditing process for research data usage reporting to be similar to audits in 
the context of the COUNTER Code of Practice Release 5, it is not yet known which organizations are willing to perform audits according to the Code of Practice for Research Data Usage Metrics, and how these audits differ from COUNTER Code of Practice Release 5 audits. For these reasons audits for research data usage reporting according to the Code of Practice for Research Data Usage Metrics are not required at this point in time.

\subsubsection{Privacy and User Confidentiality}

Statistical reports or data that reveal information about individual users will not be released or sold by content providers without the permission of that individual user, the consortium, and its member institutions (ICOLC Guidelines for Statistical Measures of Usage of Web-Based Information Resources (1998, revised 2001, 2006), 2006).

\subsubsection{Relationship to other Standards, Protocols and Codes}

The Code of Practice for Research Data Usage Metrics builds on several existing industry initiatives and standards that address content provider-based online performance measures. In addition to the COUNTER Code of Practice this includes the Scholix Metadata Schema for the Exchange of Scholarly Communication Links (Burton et al., 2017) and the NISO Alternative Assessment Metrics Project (NISO RP-25-2016: Outputs of the NISO Alternative Assessment Metrics Project, 2016).

Where appropriate, definitions of data elements and other terms from these sources have been used in this Code of Practice for Research Data Usage Metrics, and these are identified in Appendix A.

\subsection{Changes from Previous Versions}

This is the first release of the Code of Practice for Research Data Usage Metrics. 


\section{Overview}

This section provides an overview of the scope of the Code of Practice for Research Data Usage Metrics.

Section 3 Technical Implementation of Reports introduces the REQUIRED reports, describes the common format shared by all reports, and defines the report attributes and their values.

Section 4 Reports provides detailed specifications for each report. Use this section to understand what elements are included in each report.

Section 5 Delivery of Reports outlines the options a content provider MUST provide to enable customers to access their reports.

Section 6 Logging Usage describes various options used for logging usage transactions.

Section 7 Processing Rules for Underlying Data discusses topics such as which return codes to count, double-click filtering, calculating unique datasets accessed in a session, robots and internet crawlers, and machine access.

Section 8 SUSHI for Automated Report Harvesting offers a more in-depth description of the REQUIRED SUSHI support. 


\section{Technical Specifications for Reports}

\subsection{Reports for Libraries and Data Centers}

\subsubsection{Master Reports}

Master Reports include all relevant metrics and attributes; they are intended to be customizable through the application of filters and other configuration options, allowing users to create a report specific to their needs. The Dataset Master Report used in the Code of Practice for Research Data Usage Metrics are shown in Table 3.1, along with its Report ID, Report Name and Host Types who are expected to provide these reports (see Section 3.3.1 for details on Host Types).

Table 3.1: Master Reports

\begin{tabular}{|l|l|l|l|}
\hline Report_ID & Report_Name & Details & $\begin{array}{l}\text { Host Types } \\
\text { MUST be provided }\end{array}$ \\
\hline DSR & $\begin{array}{l}\text { Dataset Master } \\
\text { Report }\end{array}$ & $\begin{array}{l}\text { A granular customizable report } \\
\text { showing activity at the level of the } \\
\text { dataset that allows the user to apply } \\
\text { filters and select configuration } \\
\text { options. }\end{array}$ & $\begin{array}{l}\text { Repository } \\
\text { Data Repository }\end{array}$ \\
\hline
\end{tabular}

\subsection{Formats for Reports}

Code of Practice for Research Data Usage Metrics reports can be delivered in tabular form or as machine-readable JSON file via the SUSHI protocol. The tabular form MUST be a tabseparated-value Unicode text file. The machine-readable format MUST comply with the Research Data SUSHI API Specification (See Section 8).

All reports have the same layout and structure. Note that the Research Data SUSHI API Specification includes the same elements with the same or similar names; therefore, understanding the tabular reports translates to an understanding of what is REQUIRED in reports retrieved via SUSHI.

All reports have a header. In tabular reports, the header is separated from the body with a blank row. Beneath that is the body of the report with column headings. The contents of the body will vary by report. All of this is discussed in more detail below. 


\subsubsection{Report Header}

The first 10 rows of a tabular report contain the header, and the 11th row is always blank. The COUNTER Code of Practice Release 5 rows Institution_Name and Institution_ID are not used. The header information is presented as a series of name-value pairs, with the names appearing in Column A and the corresponding values appearing in Column B. All tabular reports have the same names in Column A. Column B entries will vary by report.

Table 3.2: Report Header Elements

\begin{tabular}{|c|c|c|}
\hline Element Name & Description of value to provide & Example \\
\hline Report_Name & $\begin{array}{l}\text { The name of the report as it appears in } \\
\text { Sections } 3.1 \text { and } 3.2 \text { of this document. } \\
\text { Must be Dataset Report. }\end{array}$ & Dataset Report \\
\hline Report_ID & $\begin{array}{l}\text { The unique identifier for the reports that } \\
\text { is used in SUSHI requests. }\end{array}$ & dsr-12hd-zt65 \\
\hline Release & $\begin{array}{l}\text { The Code of Practice for Research Data } \\
\text { Usage Metrics release this report } \\
\text { complies with. Must be RD1. }\end{array}$ & RD1 \\
\hline Metric_Types & $\begin{array}{l}\text { A semicolon-space ("; ") delimited list of } \\
\text { metric types requested for this report. } \\
\text { Note that even though a Metric Type } \\
\text { was requested, it might not be included } \\
\text { in the body of the report if no report } \\
\text { items had usage of that type. }\end{array}$ & $\begin{array}{l}\text { Unique_Dataset_Investigations; } \\
\text { Unique_Dataset_Requests }\end{array}$ \\
\hline Report_Filters & $\begin{array}{l}\text { A series of zero or more report filters } \\
\text { applied on the reported usage, excluding } \\
\text { metric types (which appear in a separate } \\
\text { row). Typically, a report filter affects the } \\
\text { amount of usage reported. Entries } \\
\text { appear in the form of } \\
\text { "filter_Name=filter_Value” with multiple } \\
\text { filter name-value pairs separated with a } \\
\text { semicolon-space (“; ") and multiple filter } \\
\text { values for a single filter name separated }\end{array}$ & $\begin{array}{l}\text { Access_Method=Regular; } \\
\text { Access_Method=Machine }\end{array}$ \\
\hline
\end{tabular}




\begin{tabular}{|c|c|c|}
\hline & by the vertical pipe ("l”) character. & \\
\hline Report_Attributes & $\begin{array}{l}\text { A series of zero or more report attributes } \\
\text { applied to the report. Typically, a report } \\
\text { attribute affects how the usage is } \\
\text { presented but does not change the } \\
\text { numbers. } \\
\text { Entries appear in the form of } \\
\text { "attribute_name=attribute_value" with } \\
\text { multiple attribute name-value pairs } \\
\text { separated with a semicolon-space ("; ") } \\
\text { and multiple attribute values for a single } \\
\text { attribute name separated by the vertical } \\
\text { pipe ("|") character. }\end{array}$ & Attributes_To_Show=Access_Methoc \\
\hline Exceptions & $\begin{array}{l}\text { An indication of some difference } \\
\text { between the usage that was created and } \\
\text { the usage that is being presented in the } \\
\text { report. The format for the exception } \\
\text { values are: "Error_No: } \\
\text { Exception_Description" (Data). The } \\
\text { Error_No and Exception_Description } \\
\text { MUST match values provided in Table } \\
\text { B.1 of Appendix B. The data is } \\
\text { OPTIONAL. } \\
\text { Note that for tabular reports, only the } \\
\text { limited set of exceptions where usage is } \\
\text { returned will apply. }\end{array}$ & $\begin{array}{l}\text { 3040: Partial Data Returned (request } \\
\text { was for 2016-01-01 to 2016-12-31; } \\
\text { however, usage is only available to } \\
\text { 2016-08-30). } \\
\text { 3040: Partial Data Returned }\end{array}$ \\
\hline Reporting_Period & $\begin{array}{l}\text { The date range for the usage } \\
\text { represented in the report, in the form of: } \\
\text { "begin_date=yyyy-mm-dd"; } \\
\text { "end_date=yyyy-mm-dd". Should } \\
\text { conform with ISO } 8601 \text { (ISO } 8601: 2004 \\
\text { - Data elements and interchange } \\
\text { formats, 2004). } \\
\text { The begin_date MUST be the first day }\end{array}$ & $\begin{array}{l}\text { begin_date=2016-01-01; } \\
\text { end_date=2016-08-30 }\end{array}$ \\
\hline
\end{tabular}




\begin{tabular}{|l|l|l|}
\hline & $\begin{array}{l}\text { of the month, whereas the end_date can } \\
\text { be the last day of the month for a } \\
\text { complete monthly report, or any other } \\
\text { day in the month for a partial monthly } \\
\text { report (See Section 3.3.7) }\end{array}$ & \\
\hline Created & $\begin{array}{l}\text { The date the usage was prepared, in the } \\
\text { form of "yyyy-mm-dd" according to ISO } \\
8601 \text { (ISO 8601:2004 - Data elements } \\
\text { and interchange formats, 2004). }\end{array}$ & 2010-11 \\
\hline Created_By & $\begin{array}{l}\text { The name of the organization or system } \\
\text { that created the report }\end{array}$ & DataONE \\
\hline (blank row) & Row 11 MUST be blank & \\
\hline
\end{tabular}

\subsection{Report Common Attributes and Elements}

COUNTER Code of Practice Release 5 introduced several new elements and attributes in order to help organize the information in a single, consistent, and coherent Code of Practice. The Code of Practice for Research Data Usage Metrics uses a subset of these elements and attributes relevant for research data.

\subsubsection{Host Types}

Research data sage reports are provided by different types of content hosts, and the usage reporting needs vary by host type. Although the "Host Type" does not appear on the report, the Code of Practice uses "Host Types" throughout this document to help content providers identify which reports, elements, metric types, and attributes are relevant to them.

The Code of Practice for Research Data Usage Metrics uses the following host types: 
Table 3.3: List of Host Types

\begin{tabular}{|l|l|l|}
\hline Host Type Category & Description & Example \\
\hline Repository & $\begin{array}{l}\text { A repository that hosts multiple research output } \\
\text { types including research data. Institutional } \\
\text { repositories are typically in this category. }\end{array}$ & Figshare \\
\hline Data Repository & $\begin{array}{l}\text { A research data repository hosting only research } \\
\text { data. Disciplinary repositories are typically in this } \\
\text { category. }\end{array}$ & $\begin{array}{l}\text { CDL Dash, } \\
\text { Dryad Digital } \\
\text { Repository }\end{array}$ \\
\hline
\end{tabular}

\subsubsection{Data Types}

The COUNTER Code of Practice Release 5 reports scholarly information in many ways. These major groupings are referred to as Data Types. Only the Dataset Data Types are used by the Code of Practice for Research Data Usage Metrics. Reporting of collections is restricted to preset collections that are defined like databases.

Table 3.4: List of Data Types

\begin{tabular}{|l|l|l|l|}
\hline Data Type & Description & Host Types & Reports (Abbrev) \\
\hline Dataset & A dataset & $\begin{array}{l}\text { Repository } \\
\text { Data Repository }\end{array}$ & DSR \\
\hline
\end{tabular}

\subsubsection{Metric types}

The following metric types are defined to enable reporting. There is no significant difference to the COUNTER Code of Practice Release 5.

\section{Investigations and Requests of Items and Titles}

This group of Metric Types represents activities where datasets were retrieved (Requests) or information about a dataset (e.g. metadata) was examined (Investigations). Any user activity that can be attributed to a Dataset will be considered an Investigation, including downloading or viewing the Dataset. Requests are limited to user activity related to retrieving or viewing the Dataset itself. 


\section{Total_Dataset, Unique_Dataset}

The metric types that begin with Total_mean that if a dataset was accessed multiple times in a user session, the metric would increase by the number of times the Dataset was accessed (minus any adjustments for double-clicks).

Unique_Dataset metrics help eliminate the effect different styles of user interface may have on usage counts. If the same dataset was accessed multiple times in a given user session, the corresponding metric can only increase by 1 to simply indicate that the dataset was accessed in the session.

Table 3.5: List of Metric Types

\begin{tabular}{|c|c|c|c|}
\hline Metric Type & Description & Host Type & Reports \\
\hline Total_Dataset_Investigations & $\begin{array}{l}\text { Total number of times a Dataset or } \\
\text { information related to a Dataset was } \\
\text { accessed and the data volume in } \\
\text { megabytes that was transferred. } \\
\text { Double click filters are applied to these } \\
\text { transactions. Investigations (counts } \\
\text { and volume) are reported for each } \\
\text { version of the Dataset and for the } \\
\text { cumulative total across versions. }\end{array}$ & $\begin{array}{l}\text { Repository } \\
\text { Data Repository }\end{array}$ & DSR \\
\hline Unique_Dataset_Investigations & $\begin{array}{l}\text { Number of datasets investigated in } \\
\text { unique user-sessions. If investigations } \\
\text { for multiple components of the same } \\
\text { Dataset occur in the same user- } \\
\text { session, there MUST be only one } \\
\text { "unique" activity counted for that } \\
\text { Dataset. Investigations (counts and } \\
\text { volume) are reported for each version } \\
\text { of the Dataset and for the cumulative } \\
\text { total across versions. }\end{array}$ & $\begin{array}{l}\text { Repository } \\
\text { Data Repository }\end{array}$ & DSR \\
\hline Total_Dataset_Requests & $\begin{array}{l}\text { Total number of times a Dataset was } \\
\text { retrieved (the content was accessed or } \\
\text { downloaded in full or a section of it) }\end{array}$ & $\begin{array}{l}\text { Repository } \\
\text { Data Repository }\end{array}$ & DSR \\
\hline
\end{tabular}




\begin{tabular}{|l|l|l|l|}
\hline & $\begin{array}{l}\text { and the data volume in megabytes that } \\
\text { was transferred. Double-click filters } \\
\text { applied. Requests (counts and volume) } \\
\text { are reported for each version of the } \\
\text { Dataset and for the cumulative total } \\
\text { across versions. }\end{array}$ & DSR \\
\hline Unique_Dataset_Requests & $\begin{array}{l}\text { Number and data volume of Datasets } \\
\text { requested in unique user-sessions. If } \\
\text { requests for multiple components of } \\
\text { the same Dataset occur in the same } \\
\text { user-session, there MUST be only one } \\
\text { "unique" activity counted for that } \\
\text { Dataset. Requests (counts and } \\
\text { volume) are reported for each version } \\
\text { of the Dataset and for the cumulative } \\
\text { total across versions. }\end{array}$ & Data Repository & Repository \\
\hline
\end{tabular}

\subsubsection{Access Methods}

In order to track content usage by machines, and to keep that usage separate from regular usage by humans, the Access_Method attribute is used.

Table 3.6: List of Access Methods

\begin{tabular}{|l|l|l|l|}
\hline Access_Method & Description & Host Type & Reports \\
\hline Regular & $\begin{array}{l}\text { Refers to activities on a platform or content host } \\
\text { that represent typical user behavior. }\end{array}$ & $\begin{array}{l}\text { Repository } \\
\text { Data Repository }\end{array}$ & DSR \\
\hline Machine & $\begin{array}{l}\text { Refers to activities on a platform or content host } \\
\text { that represent typical machine behavior. This } \\
\text { includes only legitimate machine access and } \\
\text { excludes internet robots and crawlers (see } \\
\text { Section 7.8). }\end{array}$ & $\begin{array}{l}\text { Rata Repository } \\
\text { Datory }\end{array}$ & DSR \\
\hline
\end{tabular}




\subsubsection{Year of Publication (YOP)}

Analyzing collection usage by the age of the content is also desired. The "YOP" usage attribute represents year of publication.

Table 3.7: Year of Publication Formatting

\begin{tabular}{|l|l|l|l|}
\hline YOP & Description & Host Type & Reports \\
\hline yyyy & $\begin{array}{l}\text { The Year of Publication for the item as a four-digit } \\
\text { year. If the year of publication is not known, use a } \\
\text { value of } 0001 .\end{array}$ & $\begin{array}{l}\text { Repository } \\
\text { Data Repository }\end{array}$ & DSR \\
\hline
\end{tabular}

\subsubsection{Partial Monthly Reports}

The Reporting_Period can end before the last day of the month, in which case the report for that month will be partial. This enables incremental updates of usage reporting during the course of a month. These incremental updates always replace the previous report for that month. Reporting of usage broken down by day is not supported in this release of the Code of Practice for Research Data Usage Metrics.

\subsubsection{Zero Usage}

Inclusion of zero-usage reporting for everything, including unsubscribed content, could make reports unmanageably large.

- For tabular reports

○ Omit any row where the Reporting Period Total would be zero.

- If the Reporting Period Total is $>0$, but usage for an included month is zero, set the cell value for that month to 0 .

- For SUSHI version of reports

O Omit any Instance element with a count of zero.

- Omit Performance elements that don't have at least one Instance element.

○ Omit ReportItems elements that don't have at least one Performance element. 


\subsubsection{Missing and Unknown Field Values}

- For tabular reports

- If a field value is missing or unknown (i.e. the DOI for an item doesn't exist or isn't known), the field MUST be left blank. For clarity, the field MUST NOT contain values such as "unknown" or "n/a".

- For SUSHI version of reports

- If the value of a field is missing or unknown and the Research Data SUSHI API Specification (see Section 8) indicates the field is REQUIRED, the value of the field MUST be expressed as empty as appropriate for the data type.

- If the value of a field is missing or unknown and the field is not REQUIRED according to the Research Data SUSHI API Specification, the field MUST be omitted from the response. 


\section{Reports}

\subsection{Dataset Reports}

Dataset reports provide a summary of activity related to a dataset and provide a means of evaluating the reuse of that dataset.

Table 4.1: Dataset Master Report and Standard Views

\begin{tabular}{|l|l|l|l|}
\hline Report_ID & Report_Name & Details & Host Types \\
\hline DSR & $\begin{array}{l}\text { Dataset Master } \\
\text { Report }\end{array}$ & $\begin{array}{l}\text { A granular, customizable report showing activity } \\
\text { at the level of the Dataset that allows the user to } \\
\text { apply filters and select other configuration } \\
\text { options. }\end{array}$ & $\begin{array}{l}\text { Repository } \\
\text { Data Repository }\end{array}$ \\
\hline
\end{tabular}

\subsubsection{Report Header}

Table 4.2 shows the header details for the Dataset Master Report which contain additional filters and breakdowns beyond those included in the standard COUNTER reports, and are reported at the Dataset level, and its Standard Views.

For the tabular reports, elements MUST appear in the exact order shown, and spelling, casing and punctuation of labels (Column A) and fixed data elements such as report names (Column B) MUST match exactly. The SUSHI version of the report MUST comply with the Report_Header definition in the Research Data SUSHI API Specification (see Section 8). Entries in the table appearing in italics describe the values to include.

Table 4.2: Header for Dataset Master Report and Standard Views

\begin{tabular}{|l|l|l|}
\hline Row & $\begin{array}{l}\text { Label for Tabular Report } \\
\text { (column A) }\end{array}$ & $\begin{array}{l}\text { Value for Tabular Report } \\
\text { (column B) }\end{array}$ \\
\hline 1 & Report_Name & Dataset Master Report \\
\hline 2 & Report_ID & DSR \\
\hline 3 & Release & RD1 \\
\hline 4 & Metric_Types & $\begin{array}{l}\text { Semicolon-space delimited list of metric types included in the } \\
\text { report }\end{array}$ \\
\hline
\end{tabular}




\begin{tabular}{|c|c|c|}
\hline 5 & Report_Filters & $\begin{array}{l}\text { Semicolon-space delimited list of filters applied to the data to } \\
\text { generate the report }\end{array}$ \\
\hline 6 & Report_Attributes & $\begin{array}{l}\text { Semicolon-space delimited list of report attributes applied to } \\
\text { the data to generate the report }\end{array}$ \\
\hline 7 & Exceptions & $\begin{array}{l}\text { Any exceptions that occurred in generating the report, in the } \\
\text { format "Error_Number: Error_Description" }\end{array}$ \\
\hline 8 & Reporting_Period & $\begin{array}{l}\text { Date range requested for the report in the form of "yyyy-mm- } \\
\text { dd" to "yyyy-mm-dd". The "dd" of the from-date is } 01 \text {. The "dd" } \\
\text { of the to-date can be the last day of the to- month, or another } \\
\text { day of the to-month, in which case the reporting with be partia } \\
\text { for that month. }\end{array}$ \\
\hline 9 & Created & Date the report was run in the format of "yyyy-mm-dd" \\
\hline 10 & Created_By & Name of organization or system that generated the report \\
\hline 11 & (blank) & (blank) \\
\hline
\end{tabular}

\subsubsection{Column Headings/Elements}

When applicable, the following elements MUST appear in the tabular report in the order they appear in Table 4.3. For guidance on how these fields appear in the JSON format, refer to the Research Data SUSHI API Specification (see Section 8).

Table 4.3: Column Headings/Elements for Dataset Master Report and Standard Views

\begin{tabular}{|l|l|}
\hline Field Name (Tabular) & DSR \\
\hline Dataset_Title & $\mathrm{M}$ \\
\hline Publisher & $\mathrm{M}$ \\
\hline Publisher_ID & $\mathrm{M}$ \\
\hline Creators & $\mathrm{O}$ \\
\hline Publication_Date & $\mathrm{O}$ \\
\hline Dataset_Version & $\mathrm{O}$ \\
\hline
\end{tabular}




\begin{tabular}{|l|l|}
\hline DOI & $\mathrm{M}^{*}$ \\
\hline Other_ID & $\mathrm{M}^{*}$ \\
\hline URI & $\mathrm{M}^{*}$ \\
\hline YOP & $\mathrm{O}$ \\
\hline Access_Method & $\mathrm{O}$ \\
\hline Metric_Type & $\mathrm{M}$ \\
\hline Reporting_Period_Total & $\mathrm{M}$ \\
\hline mmm-yyyy & $\mathrm{M}$ \\
\hline
\end{tabular}

* The tabular report MUST either include DOI, OTHER_ID or URL.

\subsubsection{Filters and Attributes}

Table 4.4 presents the values that can be chosen for the Dataset Master Report and that are preset for the Standard Views. 
Table 4.4: Filters/Attributes for Item Master Report and Standard Views

\begin{tabular}{|c|c|}
\hline Filter/Attribute & DSR \\
\hline YOP & $\begin{array}{l}\text { All years, a specific year, or a range of years. Use } \\
\text { "0001" for unknown. }\end{array}$ \\
\hline Access_Method & $\begin{array}{l}\text { One or all of: } \\
\text { - Regular } \\
\text { - Machine }\end{array}$ \\
\hline Version & Either "All" or a specific version, e.g. "1.3". \\
\hline Metric_Type & $\begin{array}{l}\text { One or more of: } \\
\text { - Total_Dataset_Investigations } \\
\text { - Total_Dataset_Requests } \\
\text { - Unique_Dataset_Investigations } \\
\text { - Unique_Dataset_Requests }\end{array}$ \\
\hline Exclude_Monthly_Details & Either "True" or "False". \\
\hline
\end{tabular}

If a filter is applied to a column that doesn't show on the report, usage for all selected attribute values is summed and the totals are presented in the report. 


\section{Delivery of Reports}

Content providers MUST make tabular versions of reports available from an administrative/reporting site. All reports provided by the content provider MUST also be available via SUSHI protocols. Delivery requirements are:

- Reports MUST be provided in both of the following formats:

- Tab Separated Value (TSV) file that can be easily imported into spreadsheet programs such as Microsoft Excel without loss or corruption of data.

- JSON formatted in accordance with the Research Data SUSHI API Specification (Research Data SUSHI API Specification, 2018).

- Each report MUST be delivered as a separate file to facilitate automated processing of usage reports.

- Tabular reports MUST be made available through a website.

- The website MAY be password-controlled.

$\circ$ Email alerts MAY be sent when data is updated.

- The report interface MUST provide filter and configuration options for the Master Reports that apply to the content provider.

- The report interface MUST offer all Standard Views that apply to the content provider's host type(s) and Standard View options MUST automatically apply the REQUIRED filter and configuration options and not allow the user to alter the filters or configuration options except for the usage begin and end dates.

- The date range fields on the user interface SHOULD default to the latest month with complete usage. For example, if the current date is 15 May 2019 and April usage has been processed, the begin date would default to 01 April 2019 and the end date would default to 30 April 2019. If the April usage has not yet been processed, the start and end dates would default to 01 March 2019 to 31 March 2019. If the May usage has already been processed partially, the begin date would default to 01 April 2019 and the end date would default to the date until which usage has been processed, e.g. 10 May 2019.

○ Master Reports must include the option to Exclude_Monthly_Details. When selected, the monthly columns are excluded from the report (only

ReportingPeriod Totals appear). Note: this option is NOT available for reports 
retrieved via SUSHI; however, SUSHI does offer a Granularity Report Attribute that allows usage to be retrieved with a granularity of month, year, or totals.

- Reports MUST be provided monthly.

- Data MUST be updated within 1 month of the end of the reporting period.

- Usage MAY be processed for the entire month before usage for that month is included in reports. If usage for a full given month is not yet available, partial usage for that month MAY be returned.

- A minimum of the current year plus the prior most recent 24 months of usage data MUST be available, or the period that reports have been generated according to the Code of Practice for Research Data Usage Metrics if that period is shorter than 24 months.

- The reports MUST allow the customer the flexibility to specify a date range, in terms of months, within the most recent 24-month period. Where no date range is specified, the default MUST be calendar year and calendar-year-to-date reports for the current year.

- Reports MUST be available for harvesting via the SUSHI protocol within 1 month of the end of the reporting period. 


\section{Logging Usage}

Usage data can be generated in a number of ways, with two common approaches:

- Log file analysis, which reads log files containing web server transaction records

- Page tagging, which uses JavaScript to notify a third-party server when a page is rendered by a web browser.

Each of these approaches has advantages and disadvantages, summarized below.

\subsection{Log File Analysis}

Advantages of log file analysis over page tagging include:

- Web servers normally produce log files, so the raw data are already available. No changes to the website are required.

- The data is on the organization's own servers in a standard rather than proprietary format. This makes it easy for an organization to switch analysis programs later, use several different programs, and analyze historical data with a new program.

- Log files contain information on visits from search engine spiders. Although these MUST NOT be reported as part of user activity, it is useful information for search engine optimization.

- Log files require no additional DNS lookups. Thus, there are no external server calls which can slow page load speeds or result in uncounted page views.

- The web server reliably records every transaction it makes, including items such as content generated by scripts, and does not rely on the visitor's browser.

\subsection{Page Tagging}

The main advantages of page tagging over log file analysis are:

- Counting is activated by opening the page, not requesting it from the server. If a page is cached it will not be counted by the server. Cached pages can account for a significant proportion of page views.

- Data is gathered via a component (tag) in the page, usually written in JavaScript, in conjunction with a server backend to manipulate and store the data in a database allowing complete control over how the data is represented. 
- The script may have access to additional information on the web client user that was not sent in the query.

- Page tagging can report on events that do not involve a request to the web server.

- Page tagging is available to companies who do not have access to their own web servers.

- The page-tagging service manages the process of assigning cookies to visitors; with log file analysis, the server must be configured to do this.

- Log file analysis is almost always performed in-house. Page tagging can be done inhouse but is more often provided as a third-party service. The cost differences between these two models can also be a consideration. 


\section{Processing Rules for Underlying Reporting Data}

Usage data for usage report generation should ensure that only intended usage is recorded and that all requests not intended by the user are excluded.

Because the way usage records are generated can differ across platforms, it is impractical to describe all the possible filters and techniques used to clean up the data. This Code of Practice therefore specifies only the requirements to be met by data used for building usage reports.

\subsection{Return codes}

Return codes in this Code of Practice for Research Data Usage Metrics are not different from the specifications in the COUNTER Code of Practice Release 5. Successful and valid requests MUST be counted. Successful requests are those with specific HTTP status codes indicating successful retrieval of the content (200 and 304). HTTP status codes are defined and maintained by IETF (Fielding \& Reschke, 2014).

\subsection{Double-click Filtering}

The intent of double-click filtering is to prevent over-counting which may occur when a user clicks the same link multiple times in succession, e.g. when frustrated by a slow internet connection. Double-click filtering applies to all metric types. The double-click filtering rule is as follows:

A "double-click" is defined as repeated access to a web accessible resource by the same user within a session, within a time period. Double-clicks on a link by the same user within a 30second period MUST be counted as one action. For the purposes of the Code of Practice for Research Data Usage Metrics, the time window for a double-click on any page is set at a maximum of 30 seconds between the first and second mouse clicks. For example, a click at 10.01.00 and a second click at 10.01.29 would be considered a double-click (one action); a click at 10.01.00 and a second click at 10.01.35 would count as two separate single clicks (two actions).

A double-click may be triggered by a mouse-click or by pressing a refresh or back button. When two actions are made for the same URL within 30 seconds the first request MUST be removed and the second retained. 
Any additional requests for the same URL within 30 seconds (between clicks) MUST be treated identically: always remove the first and retain the second.

There are different ways to track whether two requests for the same URL are from the same user and session. These options are listed in order of increasing reliability, with Option 4 being the most reliable.

1. If the user is identified only through their IP address, that IP combined with the browser's user-agent (presented in the HTTP header) MUST be used to trace double-clicks.

Multiple users on a single IP address with the same browser user-agent can occasionally lead to separate clicks from different users being logged as a double-click from one user. This will only happen if the multiple users are clicking on exactly the same content within a few seconds of each other. One-hour slices MUST be used as sessions.

2. When a session cookie is implemented and logged, the session cookie MUST be used to identify double-clicks.

3. When a user cookie is available and logged, the user cookie MUST be used to identify double-clicks.

4. When an individual has logged in with their own profile, their username MUST be used to trace double-clicks.

\subsection{Counting Unique Datasets}

Some metric types count the number of unique items that had a certain activity, such as a

\section{Unique_Dataset_Requests or Unique_Dataset_Investigations.}

For the purpose of metrics, a dataset is the typical unit of content being accessed by users. The dataset MUST be identified using a unique identifier such as a DOI, regardless of format.

The rules for calculating the unique dataset counts are as follows:

Multiple activities qualifying for the metric type in question representing the same dataset and occurring in the same user-sessions MUST be counted as only one "unique" activity for that dataset.

A "User Session" is defined as activity by a user in a period of one hour. It may be identified in any of the following ways: by a logged session ID + transaction date, by a logged user ID (if users log in with personal accounts) + transaction date + hour of day (day is divided into 24 one- 
hour slices), by a logged user cookie + transaction date + hour of day, or by a combination of IP address + user agent + transaction date + hour of day.

To allow for simplicity in calculating User Sessions when a session ID is not explicitly tracked, the day will be divided into 24 one-hour slices and a surrogate session ID will be generated by combining the transaction date + hour time slice + one of the following: user ID, cookie ID, or IP address + user agent. For example, consider the following transaction:

- Transaction date/time: 2017-06-15 13:35

- IP address: 192.1.1.168

- User agent: Mozilla/5.0

- Generated session ID: 192.1.1.168|Mozilla/5.0|2017-06-15|13

The above surrogate session ID does not provide an exact analogy to a session. However, statistical studies show that the result of using such a surrogate session ID results in unique counts are within $1-2 \%$ of unique counts generated with actual sessions.

\subsection{Attributing Usage when Item Appears in More Than One Database}

Content providers that offer databases where a given dataset is included in multiple databases MUST attribute the Investigations and Requests metrics to just one database. They could use a consistent method of prioritizing databases or pick the database randomly.

\subsection{Internet Robots and Crawlers}

The intent is to exclude web robots and spiders but include usage by humans accessing content through a scripting language or automated tool, whether interactively or standalone.

Web robots and crawlers intended for search indexing and related applications SHOULD be excluded via the application of a blacklist of known user agents for these robots. This blacklist MUST NOT include general purpose user agents that are commonly used by researchers (e.g., python, curl, wget, and Java), and the blacklist will be maintained as a subset of the COUNTER Code of Practice Release 5 list of internet robots and crawlers (COUNTER-Robots, 2017).

Generally, user agents reflecting programmatic access to specific datasets will not be included in the blacklist.

Usage counts by scripted and automated processes MUST NOT be excluded unless they can demonstrably be shown to originate from a blacklisted agent, such as an IP address of a known 
search agent. New or unknown user agents SHOULD be counted unless there is demonstrable evidence that they represent solely a web indexing agent.

\subsection{Machine Access}

Many researchers access and analyze data using scripts or automated tools, especially large data sets, and excluding those uses would be inaccurate and bias the counts. The Access_Method of type Machine is used to distinguish this kind of access.

\subsubsection{Principles for reporting usage}

- The Code of Practice for Research Data Usage Metrics does not record machine use itself, as most of this activity takes place after a dataset has been downloaded. All we can do is track the count of datasets downloaded using machines.

- Usage associated with machine access activity MUST be tracked by assigning an Access_Method of Machine.

- Usage associated with machine activity MUST be reported using the Dataset Master Report by identifying such usage as “Access_Method=Machine".

\subsubsection{Detecting machine activity}

For the purpose of reporting usage according to the Code of Practice for Research Data Usage Metrics, machine access does not require prior permission and/or the use of specific endpoints or protocols. This is in contrast to the COUNTER Code of Practice Release 5.

The distinction between legitimate machine use and robot or web crawler traffic is made based on the user agent (see Section 7.5). 


\section{SUSHI for Automated Report Harvesting}

Content providers MUST support automatic harvesting of reports via the SUSHI protocol as described in the NISO SUSHI Protocol (ANSI/NISO Z39.93-2014: Standardized Usage Statistics Harvesting Initiative (SUSHI) Protocol, 2014) and the Research Data SUSHI API Specification (Research Data SUSHI API Specification, 2018).

\subsection{Research Data SUSHI API Paths to Support}

The following paths (methods) MUST be supported:

Table 8.1: Research Data SUSHI API Paths

\begin{tabular}{|l|l|}
\hline Path & Description \\
\hline GET /status & $\begin{array}{l}\text { Returns the current status of the Research Data SUSHI API service. This path } \\
\text { returns a message that includes the operating status of the API, the URL to the } \\
\text { service's entry in the Register of Compliant Content Providers, and an array of } \\
\text { service alerts (if any). }\end{array}$ \\
\hline GET /reports & $\begin{array}{l}\text { Returns a list of reports supported by the Research Data SUSHI API service. } \\
\text { The response includes an array of reports, including the report identifier, the } \\
\text { release number, the report name, a description, a list of supported report filters, } \\
\text { and a list of supported report attributes. }\end{array}$ \\
\hline $\begin{array}{l}\text { GET } \\
\text { /reports/\{ReportID\} }\end{array}$ & $\begin{array}{l}\text { Each supported report has its own path, e.g. GET /reports/DSR for dataset } \\
\text { requests. }\end{array}$ \\
\hline
\end{tabular}

\subsection{Authentication and Security for the Research Data SUSHI API}

The Research Data SUSHI API SHOULD be implemented using TLS (HTTPS). The Research Data SUSHI API MAY be secured using an API key or username/password assigned to the organization harvesting the usage.

\subsection{Report Filters and Report Attributes}

The Research Data SUSHI API Specification allows report responses to be customized to the caller's needs using report filters and report attributes. These filters and attributes are implicit for Standard Views. Filters and attributes are explicitly included as parameters on the Research Data 
SUSHI API request for Master Reports. Refer to (Research Data SUSHI API Specification, 2018) for the list of filters and attributes supported by the various reports.

\subsection{Research Data SUSHI API Errors and Exceptions}

Implementations of the Research Data SUSHI API MUST comply with the warnings, exceptions and errors described in the Research Data SUSHI API Specification. See Appendix B.

\subsection{SUSHI Service Limits}

The content provider MUST NOT place limits on the SUSHI service (such as requests per day or amount of data transferred) that would prevent users from retrieving reports. 


\section{Acknowledgements}

The authors want to thank Tasha Mellins-Cohen, Amber Budden, Kristian Garza and Christopher Jones for helpful discussions of the manuscript.

\section{References}

ANSI/NISO Z39.93-2014: Standardized Usage Statistics Harvesting Initiative (SUSHI) Protocol. (2014). Retrieved from http://www.niso.org/standards-committees/sushi

Burton, A., Fenner, M., Haak, W., \& Manghi, P. (2017). Scholix Metadata Schema for Exchange of Scholarly Communication Links. Zenodo. https://doi.org/10.5281/zenodo.1120265

Costas, R., Meijer, I., Zahedi, Z., \& Wouters, P. (2013). The Value of Research Data-Metrics for datasets from a cultural and technical point of view. A Knowledge Exchange Report. Retrieved from http://www.knowledge-exchange.info/event/value-reseach-data-metrics

COUNTER Code of Practice Release 5. (2017). Retrieved from

https://www.projectcounter.org/code-of-practice-five-sections/abstract/

COUNTER-Robots: Official list of user agents that are regarded as robots/spiders by

COUNTER. (2017). Retrieved from https://github.com/atmire/COUNTER-Robots

Dekkers, A., \& Isaac, A. (2018). Data Catalog Vocabulary (DCAT) 1.1. Retrieved from

https://w3c.github.io/dxwg/dcat/

Fielding, R., \& Reschke, J. (2014). RFC7231: Hypertext Transfer Protocol (HTTP/1.1):

Semantics and Content. Retrieved from https://tools.ietf.org/html/rfc7231

ICOLC Guidelines for Statistical Measures of Usage of Web-Based Information Resources

(1998, revised 2001, 2006). (2006). Retrieved from http://icolc.net/statement/guidelines-

statistical-measures-usage-web-based-information-resources-1998-revised-2001-0

ISO 8601:2004 - Data elements and interchange formats. (2004). Retrieved from

https://www.iso.org/standard/40874.html

Kratz, J. E., \& Strasser, C. (2015). Making data count. Scientific Data, 2, 150039.

https://doi.org/10.1038/sdata.2015.39

Make Data Count. (2017). Retrieved from https://makedatacount.org/

NISO RP-25-2016: Outputs of the NISO Alternative Assessment Metrics Project. (2016).

Retrieved from http://www.niso.org/publications/niso-rp-25-2016-outputs-niso-alternativeassessment-metrics-project 
Project COUNTER. (2002). Retrieved from https://www.projectcounter.org/ Research Data SUSHI API Specification. (2018). Retrieved from https://app.swaggerhub.com/apis/COUNTER/researchdata-sushi_1_0_api/1.0.0 RFC 2119: Key words for use in RFCs to Indicate Requirement Levels. (1997). Retrieved from https://www.ietf.org/rfc/rfc2119.txt 


\section{Appendix A: Glossary of Terms}

Aligned as much as possible with the COUNTER Code of Practice Release 5 glossary.

\begin{tabular}{|c|c|}
\hline Abstract & See Description. \\
\hline Access_Method & $\begin{array}{l}\text { A COUNTER attribute indicating whether the usage related to } \\
\text { investigations and requests was generated by a human user } \\
\text { browsing and searching a website (Regular) or by a computer } \\
\text { (Machine). }\end{array}$ \\
\hline Author(s) & See Creator \\
\hline Collection & A curated collection of metadata about content items. \\
\hline Component & $\begin{array}{l}\text { A uniquely identifiable constituent part of a content item composed of } \\
\text { more than one file (digital object). }\end{array}$ \\
\hline Content item & $\begin{array}{l}\text { A generic term describing a unit of content accessed by a user of a } \\
\text { content host. Typical content items include articles, books, chapters, } \\
\text { datasets, multimedia, etc. }\end{array}$ \\
\hline Content provider & $\begin{array}{l}\text { An organization whose function is to commission, create, collect, } \\
\text { validate, host, distribute, and trade information in electronic form. }\end{array}$ \\
\hline Creator(s) & $\begin{array}{l}\text { The person/people who wrote/created the datasets whose usage is } \\
\text { being reported- }\end{array}$ \\
\hline Data repository & A content provider that provides access to research data. \\
\hline Data type & $\begin{array}{l}\text { The field identifying type of content. The Code of Practice for } \\
\text { Research Data Usage Metrics only recognizes the Data type } \\
\text { Dataset. }\end{array}$ \\
\hline Dataset & $\begin{array}{l}\text { An aggregation of data, published or curated by a single agent, and } \\
\text { available for access or download in one or more formats, with } \\
\text { accompanying metadata. Other term: data package. }\end{array}$ \\
\hline Description & $\begin{array}{l}\text { A short description of a dataset. Accessing the description falls into } \\
\text { the usage category of Investigations. }\end{array}$ \\
\hline DOI (digital object identifier) & $\begin{array}{l}\text { The digital object identifier is a means of identifying a piece of } \\
\text { intellectual property (a creation) on a digital network, irrespective of } \\
\text { its current location (IDF). }\end{array}$ \\
\hline Double-click & $\begin{array}{l}\text { A repeated click or repeated access to the same resource by the } \\
\text { same user within a period of } 30 \text { seconds. COUNTER requires that } \\
\text { double-clicks must be counted as a single click. }\end{array}$ \\
\hline Host types & $\begin{array}{l}\text { A categorization of Content Providers used by COUNTER. The Code } \\
\text { of Practice for Research Data Usage Metrics uses the following host } \\
\text { types: } \\
\text { - Repository } \\
\text { - Data Repository }\end{array}$ \\
\hline
\end{tabular}




\begin{tabular}{|c|c|}
\hline Internet robot, crawler, spider & $\begin{array}{l}\text { An identifiable, automated program or script that visits websites and } \\
\text { systematically retrieves information from them, often to provide } \\
\text { indexes for search engines rather than for research. Not all } \\
\text { programs or scripts are classified as robots. }\end{array}$ \\
\hline Investigation & $\begin{array}{l}\text { A category of COUNTER metric types that represent a user } \\
\text { accessing information related to a dataset (i.e. a description or } \\
\text { detailed descriptive metadata) or the content of the dataset itself. }\end{array}$ \\
\hline Log file analysis & $\begin{array}{l}\text { A method of collecting usage data in which the web server records } \\
\text { all of its transactions. }\end{array}$ \\
\hline Machine & $\begin{array}{l}\text { A category of COUNTER Metric Types that represents a machine } \\
\text { accessing content, e.g. a script written by a researcher. This does } \\
\text { not include robots, crawlers and spiders. }\end{array}$ \\
\hline Master reports & $\begin{array}{l}\text { Reports that contain additional filters and breakdowns beyond those } \\
\text { included in the standard COUNTER reports. }\end{array}$ \\
\hline Metadata & $\begin{array}{l}\text { A series of textual elements that describes a content item but does } \\
\text { not include the item itself. For example, metadata for a dataset } \\
\text { would typically include publisher, a list of names and affiliations of } \\
\text { the creators, the title and description, and keywords or other subject } \\
\text { classifications. }\end{array}$ \\
\hline Metric types, Metric_Type & $\begin{array}{l}\text { An attribute of COUNTER usage that identifies the nature of the } \\
\text { usage activity. }\end{array}$ \\
\hline $\begin{array}{l}\text { ORCID (Open Researcher and } \\
\text { Contributor ID) }\end{array}$ & $\begin{array}{l}\text { An international standard identifier for individuals (i.e. authors) to use } \\
\text { with their name as they engage in research, scholarship, and } \\
\text { innovation activities. }\end{array}$ \\
\hline Persistent Identifier (PID) & $\begin{array}{l}\text { Globally unique identifier and associated metadata for research data, } \\
\text { or other entities (articles, researchers, scholarly institutions) relevant } \\
\text { in scholarly communication. }\end{array}$ \\
\hline Platform & $\begin{array}{l}\text { An interface from an aggregator, publisher, or other online service } \\
\text { that delivers the content to the user and that counts and provides the } \\
\text { COUNTER usage reports. }\end{array}$ \\
\hline Provider ID & $\begin{array}{l}\text { A unique identifier for a Content Provider and used by discovery } \\
\text { services and other content sites to track usage for content items } \\
\text { provided by that provider. }\end{array}$ \\
\hline Publication date, Publication_Date & $\begin{array}{l}\text { An optional field in COUNTER item reports and Provider Discovery } \\
\text { Reports. The date of release by the publisher to customers of a } \\
\text { content item. }\end{array}$ \\
\hline Publisher & $\begin{array}{l}\text { An organization whose function is to commission, create, collect, } \\
\text { validate, host, distribute and trade information online and/or in } \\
\text { printed form. }\end{array}$ \\
\hline Regular & $\begin{array}{l}\text { A COUNTER Access_Method. Indicates that usage was generated } \\
\text { by a human user browsing/searching a website, rather than by a } \\
\text { computer. }\end{array}$ \\
\hline
\end{tabular}




\begin{tabular}{|c|c|}
\hline $\begin{array}{l}\text { Reporting period, } \\
\text { Reporting_Period }\end{array}$ & The total time period covered in a usage report. \\
\hline Request & $\begin{array}{l}\text { A category of COUNTER Metric Types that represents a user } \\
\text { accessing the dataset content. }\end{array}$ \\
\hline Session & $\begin{array}{l}\text { A successful request of an online service. A single user connects to } \\
\text { the service or database and ends by terminating activity that is either } \\
\text { explicit (by leaving the service through exit or logout) or implicit } \\
\text { (timeout due to user inactivity). (NISO). }\end{array}$ \\
\hline SUSHI & $\begin{array}{l}\text { An international standard (Z39-93) that describes a method for } \\
\text { automating the harvesting of reports. Research Data SUSHI API } \\
\text { Specification is an implementation of this standard for harvesting } \\
\text { Code of Practice for Research Data Usage Metrics reports. }\end{array}$ \\
\hline Total_Dataset_Investigations & $\begin{array}{l}\text { A COUNTER Metric_Type that represents the number of times } \\
\text { users accessed the content of a dataset, or information describing } \\
\text { that dataset (i.e. metadata). }\end{array}$ \\
\hline Total_Dataset_Requests & $\begin{array}{l}\text { A COUNTER Metric_Type that represents the number of times } \\
\text { users requested the content of a dataset. Requests may take the } \\
\text { form of viewing, downloading, or emailing the dataset provided such } \\
\text { actions can be tracked by the content provider's server. }\end{array}$ \\
\hline Transactions & A usage event. \\
\hline Unique_Dataset_Investigations & $\begin{array}{l}\text { A COUNTER Metric Type that represents the number of unique } \\
\text { "Datasets" investigated in a user-session. }\end{array}$ \\
\hline Unique_Dataset_Requests & $\begin{array}{l}\text { A COUNTER Metric Type that represents the number of unique } \\
\text { datasets requested in a user-session. }\end{array}$ \\
\hline User & A person who accesses the online resource. \\
\hline User agent & $\begin{array}{l}\text { An identifier that is part of the HTTP/S protocol that identifies the } \\
\text { software (i.e. browser) being used to access the site. May be used } \\
\text { by robots to identify themselves. }\end{array}$ \\
\hline Version & $\begin{array}{l}\text { Multiple versions of a dataset are defined by significant changes to } \\
\text { the content and/or metadata, associated with changes in one or } \\
\text { more components. }\end{array}$ \\
\hline Year of publication & Calendar year in which a dataset is published. \\
\hline
\end{tabular}




\section{Appendix B: Handling Errors and Exceptions}

As a rule, the structure of the SUSHI response will be governed by the SUSHI schema; therefore, any error conditions that can be reported will be specified within the SUSHI response. The following is a definition from the Research Data SUSHI API Specification that shows the format of the exception.

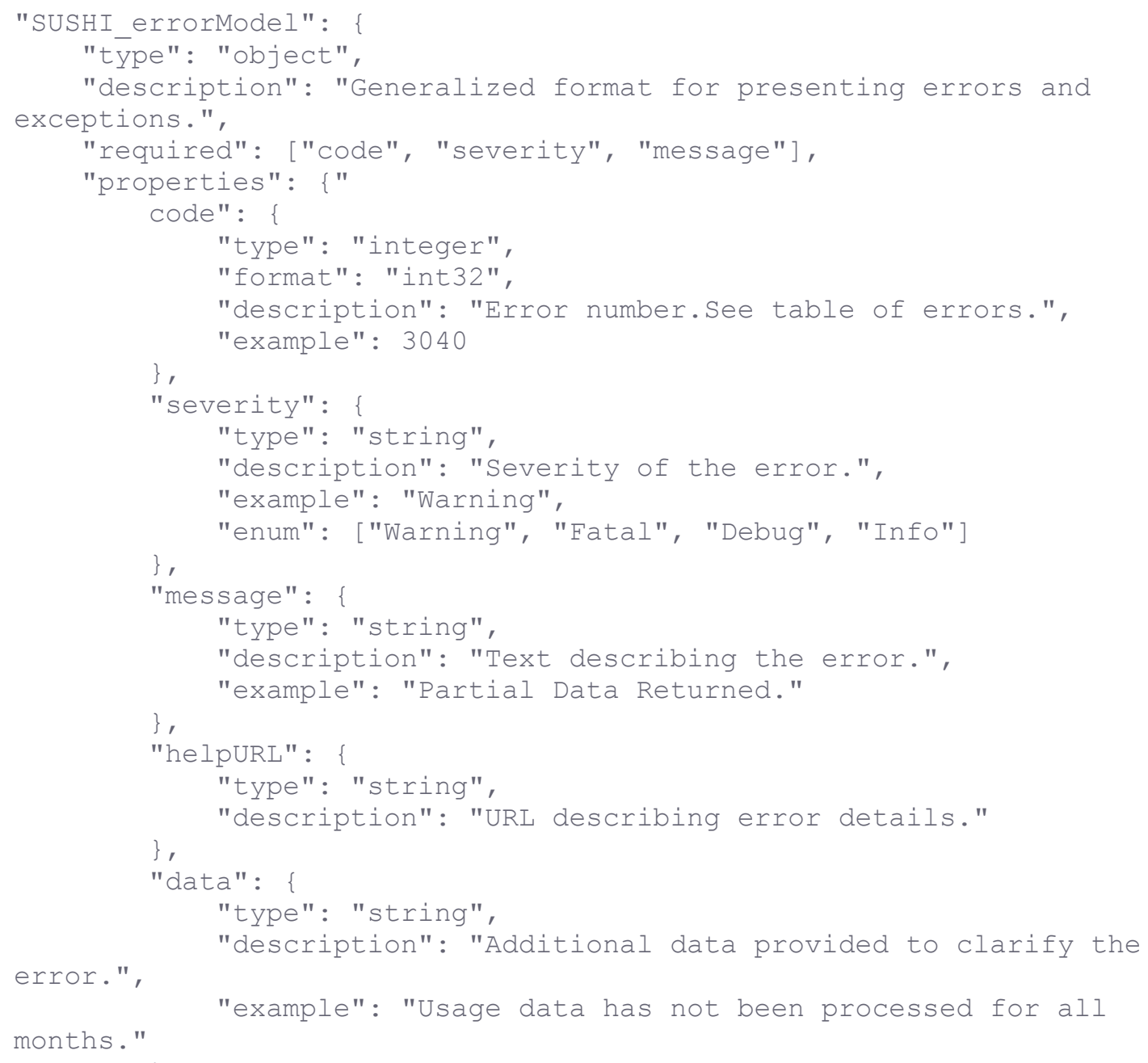

As indicated in the JSON code above, multiple exceptions can be returned, and the exceptions have the following elements:

- code: is a numeric exception number that identifies the exception. See table B.1 for values.

- severity: indicates if the exception is one of: 
- Fatal: unable to complete the transaction. The problem is with the service and may be temporary and a retry could be successful. No report is returned. Example: service busy.

- Error: unable to complete the transaction. The problem is with the request such that a retry will not be successful unless the request or other configuration details change. No report is returned. Example: Requestor not authorized.

- Warning: The transaction can be completed, but not all requested information could be returned. Examples: "Usage Not Ready for Requested Data; Partial Data Returned"

- Debug: reserved for use by developers as a means of providing additional data about the request or response to the calling application.

- message: textual description of the exception. For exception codes $>999$ the message must exactly match column 1 in table B.1.

- data: additional optional data that further describes the error. Example: for the Partial Data Returned exception, the "data" could state "You requested 2017-01-01 to 2016-1231; however, only 2017-01-01 to 2017-06-30 were available."

- helpurl: an optional variable that includes the URI to a help message that explains the exception in more detail.

Table B.1 provides a list of possible exceptions that may occur for Research Data SUSHI API reports.

Table B.1: SUSHI Exceptions

\begin{tabular}{|l|l|l|l|}
\hline $\begin{array}{l}\text { Exception } \\
\text { (message) }\end{array}$ & Severity & $\begin{array}{l}\text { Exception } \\
\text { Number } \\
\text { (code) }\end{array}$ & Invocation Conditions \\
\hline $\begin{array}{l}\text { Info or } \\
\text { Debug }\end{array}$ & $\begin{array}{l}\text { Info } \\
\text { Debug }\end{array}$ & 0 & $\begin{array}{l}\text { Any. These messages will never be standardized, and } \\
\text { service providers can design them as they see fit. }\end{array}$ \\
\hline Warnings & Warning & $1-999$ & $\begin{array}{l}\text { Any. This range is reserved for the use of service } \\
\text { providers to supply their own custom warnings. }\end{array}$ \\
\hline
\end{tabular}




\begin{tabular}{|c|c|c|c|}
\hline $\begin{array}{l}\text { Service Not } \\
\text { Available }\end{array}$ & Fatal & 1000 & $\begin{array}{l}\text { Service is executing a request, but due to internal errors } \\
\text { cannot complete the request. Service must return } \\
\text { ReportResponse and no payload. }\end{array}$ \\
\hline Service Busy & Fatal & 1010 & $\begin{array}{l}\text { Service is too busy to execute the incoming request. } \\
\text { Service must return ReportResponse with this exception } \\
\text { and no payload. Client should retry the request after some } \\
\text { reasonable time. }\end{array}$ \\
\hline $\begin{array}{l}\text { Client has made } \\
\text { too many } \\
\text { requests }\end{array}$ & Fatal & 1020 & $\begin{array}{l}\text { If the server sets a limit on the number of requests a client } \\
\text { can make within a given timeframe, the server will return } \\
\text { this error when the client exceeds that limit. The server } \\
\text { would provide an explanation of the limit in the Message } \\
\text { of the error (e.g., "Client has made too many requests. } \\
\text { This server allows only } 5 \text { requests per day per } \\
\text { RequestorID and CustomerID."). }\end{array}$ \\
\hline $\begin{array}{l}\text { Insufficient } \\
\text { Information to } \\
\text { Process Request }\end{array}$ & Fatal & 1030 & $\begin{array}{l}\text { There is insufficient data in the request to begin } \\
\text { processing (e.g., missing Requestor ID, Report is missing, } \\
\text { no Customer ID, etc.). }\end{array}$ \\
\hline $\begin{array}{l}\text { Requestor Not } \\
\text { Authorized to } \\
\text { Access Service }\end{array}$ & Error & 2000 & $\begin{array}{l}\text { If Requestor ID is not recognized or not authorized by the } \\
\text { service. }\end{array}$ \\
\hline APIKey Invalid & Error & 2020 & $\begin{array}{l}\text { The service being called requires a valid APIKey to } \\
\text { access usage data and the key provided was not valid or } \\
\text { not authorized for the data being requested. }\end{array}$ \\
\hline $\begin{array}{l}\text { Report Not } \\
\text { Supported }\end{array}$ & Error & 3000 & $\begin{array}{l}\text { The requested report name, version, or other means of } \\
\text { identifying a report that the service can process is not } \\
\text { matched against the supported reports. }\end{array}$ \\
\hline $\begin{array}{l}\text { Report Version } \\
\text { Not Supported }\end{array}$ & Error & 3010 & $\begin{array}{l}\text { Requested version of the data is not supported by the } \\
\text { service. }\end{array}$ \\
\hline $\begin{array}{l}\text { Invalid Date } \\
\text { Arguments }\end{array}$ & Error & 3020 & $\begin{array}{l}\text { Any format or logic errors involving date computations } \\
\text { (e.g., end date cannot be less than begin date). }\end{array}$ \\
\hline
\end{tabular}




\begin{tabular}{|c|c|c|c|}
\hline $\begin{array}{l}\text { No Usage } \\
\text { Available for } \\
\text { Requested Dates }\end{array}$ & Error & 3030 & Service did not find any data for the date range specified. \\
\hline $\begin{array}{l}\text { Usage Not Ready } \\
\text { for Requested } \\
\text { Dates }\end{array}$ & $\begin{array}{l}\text { Error, } \\
\text { Warning }\end{array}$ & 3031 & $\begin{array}{l}\text { Service has not yet processed the usage for one or more } \\
\text { of the requested months, if some months are available } \\
\text { that data should be returned. The exception should } \\
\text { include the months not processed in the additional data } \\
\text { element. }\end{array}$ \\
\hline $\begin{array}{l}\text { Partial Data } \\
\text { Returned }\end{array}$ & Warning & 3040 & $\begin{array}{l}\text { Request could not be fulfilled in its entirety. Data that was } \\
\text { available was returned. }\end{array}$ \\
\hline $\begin{array}{l}\text { Parameter Not } \\
\text { Recognized in } \\
\text { this Context }\end{array}$ & Warning & 3050 & $\begin{array}{l}\text { Request contained one or more parameters that are not } \\
\text { recognized by the Server in the context of the report being } \\
\text { serviced. The server should list the Name of unsupported } \\
\text { filter in the Message element of the Exception. } \\
\text { Note: The server is expected to ignore unsupported } \\
\text { parameters and continue to process the request, returning } \\
\text { data that is available without the parameter being applied. }\end{array}$ \\
\hline $\begin{array}{l}\text { Invalid } \\
\text { ReportFilter } \\
\text { Value }\end{array}$ & $\begin{array}{l}\text { Warning, } \\
\text { Error }\end{array}$ & 3060 & $\begin{array}{l}\text { Request contained one or more Filter values in the } \\
\text { ReportDefinition that are not supported by the Server. The } \\
\text { server should list the Name of unsupported filter values in } \\
\text { the Message element of the Exception. } \\
\text { Note: The server is expected to ignore unsupported filters } \\
\text { and continue to process the request, returning data that is } \\
\text { available without the filter being applied. }\end{array}$ \\
\hline $\begin{array}{l}\text { Incongruous } \\
\text { ReportFilter } \\
\text { Value }\end{array}$ & $\begin{array}{l}\text { Warning, } \\
\text { Error }\end{array}$ & 3061 & $\begin{array}{l}\text { A filter element includes multiple values in a pipe- } \\
\text { delimited list; however, the supplied values are not all of } \\
\text { the same scope. }\end{array}$ \\
\hline
\end{tabular}




\begin{tabular}{|c|c|c|c|}
\hline $\begin{array}{l}\text { Invalid } \\
\text { ReportAttribute } \\
\text { Value }\end{array}$ & $\begin{array}{l}\text { Warning, } \\
\text { Error }\end{array}$ & 3062 & $\begin{array}{l}\text { Request contained one or more ReportAttribute values in } \\
\text { the ReportDefinition that are not supported by the Server. } \\
\text { The server should list the Name of unsupported report } \\
\text { attribute values in the Message element of the Exception. } \\
\text { Note: The server is expected to ignore unsupported report } \\
\text { attributes and continue to process the request, returning } \\
\text { data that is available without the report attribute being } \\
\text { applied. }\end{array}$ \\
\hline $\begin{array}{l}\text { Required } \\
\text { ReportFilter } \\
\text { Missing }\end{array}$ & $\begin{array}{l}\text { Warning, } \\
\text { Error }\end{array}$ & 3070 & $\begin{array}{l}\text { A required filter was not included in the request. Which } \\
\text { filters are required will depend on the report and the } \\
\text { service being called. In general, the omission of a } \\
\text { required filter would be viewed as an Error; however, if } \\
\text { the service is able to process the request using a default } \\
\text { value then a Warning can be returned. The Message } \\
\text { element of the Exception should name the missing filter. }\end{array}$ \\
\hline $\begin{array}{l}\text { Required } \\
\text { ReportAttribute } \\
\text { Missing }\end{array}$ & $\begin{array}{l}\text { Warning, } \\
\text { Error }\end{array}$ & 3071 & $\begin{array}{l}\text { A required report attribute was not included in the request. } \\
\text { In general, the omission of a required filter would be } \\
\text { viewed as an Error; however, if the service is able to } \\
\text { process the request using a default value, then a Warning } \\
\text { can be returned. The Message element of the Exception } \\
\text { should name the missing filter. }\end{array}$ \\
\hline $\begin{array}{l}\text { Limit Requested } \\
\text { Greater than } \\
\text { Maximum Server } \\
\text { Limit }\end{array}$ & Warning & 3080 & $\begin{array}{l}\text { The requested value for limit (number of items to return) } \\
\text { exceeds the server limit. The server is expected to return } \\
\text { data in the response (up to the limit). The Message } \\
\text { element of the exception should indicate the server limit. }\end{array}$ \\
\hline
\end{tabular}


Note 1: An Error does not interrupt completion of the transaction (in the sense of a programmatic failure), although it may not return the expected report for the reason that is identified. A Fatal exception does not complete the transaction; the problem may be temporary and a retry could be successful.

Note 2: Optional response: Service may respond with the additional exception of Info level and include additional information in the message. For example, if the client is requesting data for a date range where the begin date is before what the service offers, the service might include a HelpURL that can provide more information about supported dates.

Note 3: If multiple exceptions are discovered, each exception should be returned in its own element. Note 4: Clarifying details about an exception (e.g., the filter that was missing or deemed invalid should be added to the Data element or Message element of the exception so that the caller knows what to correct). Note 5: If the caller gets the baseURL, the version, or method wrong, the expectation is that they will receive an HTTP 404 error since the specified path is not valid. 